\title{
Disability trajectories in activities of daily living of elderly Chinese before death
}

\author{
Wenjuan Zhang ${ }^{1}$ (D) Marcus W. Feldman ${ }^{2}$
}

Received: 11 May 2020 / Accepted: 12 August 2020 / Published online: 10 September 2020

(c) The Author(s) 2020

\begin{abstract}
This study aims to analyze the changes in activities of daily living (ADL) of the Chinese elderly before death, and to explore the heterogeneity in this process. Using data from the Chinese Longitudinal Healthy Longevity Survey (CLHLS), we quantify disability trajectories of ADL using a group-based trajectory model and find that there are three types of disability trajectory for ADL. The elderly who differ by socioeconomic status, childhood experiences, health behaviors, ages and birth cohorts show significant differences in their disability trajectories. Long duration of disability is found to be more prevalent in older females and people with high socioeconomic status. Good and stable status of ADL is more common among males and people of low socioeconomic status, while the elderly in an early cohort who died at older ages were more likely to have experienced a long duration of disability. Selective and protective effects contribute to the observed differences in trajectories.
\end{abstract}

Keywords ADL $\cdot$ Disability trajectory $\cdot$ Heterogeneity $\cdot$ Chinese elderly

\section{Introduction}

Over the last 2 decades, China has experienced accelerated population ageing and the size of the disabled older population has increased, reaching 10.84 million in 2010 , which is $6.25 \%$ of the total population of older people aged 60 and above (China Research Center on Aging 2012). Functional limitation and disability of older people (meaning they need assistance to carry out basic routine activities for

Wenjuan Zhang

zhwenj@ruc.edu.cn

Marcus W. Feldman

mfeldman@stanford.edu

1 Institute of Gerontology, School of Sociology and Population Studies, Renmin University of China, 59 Zhongguancun Ave, Haidian District, Beijing 100874, China

2 Morrison Institute for Population and Resource Studies, Stanford University, Stanford, CA 94305, USA 
survival) is a crucial consideration in planning the population's need for long-term care. Assessing the risk of functional limitation and estimating the survival duration with disability is key to estimating the burden of long-term care. However, difficulties still exist in estimating the functional capacity of Chinese older people and predicting their disability prevalence in the future (Zeng et al. 2012). Some researchers argue that the survival time with disability during later life is affected by the demographic transition (Robine and Michel 2004). At the individual level, significant heterogeneity exists in the process of older people becoming disabled (Evert et al. 2003). Instability in the decline of functional capacity also poses a challenge to prediction of the burden of long-term care and increases the difficulty of identifying those in urgent need of care.

In previous research, many indicators have been used to evaluate functional capacity of older people. Among these, physical activities of daily living (ADL) is used most frequently (Ostir et al. 1999) and includes such self-care activities as feeding, dressing, toileting, moving indoors, bathing, and continence (Katz et al. 1963). Limitation in ADL means loss of the capacity for independent living, which is also defined as disability, viewed as the gap between personal capability and environmental demand (Verbrugge and Jette 1994). The demands of the six components of ADL on physiological states of individuals are different: bathing is the most complex activity and is often the first to be limited, while feeding usually comes last (Fong and Feng 2018). Limitation only in bathing is usually identified as a mild disability and, since it is more likely to be influenced by surroundings, it is more variable. Conclusions drawn from different indicators of functional capacity are also inconsistent for reasons mentioned above. In the present study, changes in the trajectories of functional capacity will be analyzed using specific indicators.

The prevalence of disability in ADL of Chinese oldest-old has declined over the last two decades (Martin et al. 2014; Liang et al. 2015). However, some features of this decline vary over this period (Hou et al. 2018), and the patterns of decline in ADL of young-aged and middle-aged Chinese have changed differently from that of the oldest-old (Liu et al. 2009). A number of demographic features that can further influence the occurrences of death, marriage, unemployment, and other life events (Vaupel and Yashin 1985) have been ignored. As the accumulation of life events experienced by individuals, developmental trajectories show important heterogeneities. These differences have been confirmed by previous research on ADL changes of Chinese oldest-old (Gu and Zeng 2004). Zimmer et al. (2012) use a group-based trajectory model (GTM) to classify disability trajectories of ADL during the ageing process into different types and to demonstrate the heterogeneity of Chinese oldestold in changes of ADL.

Research on the change process of ADL of older people usually takes age as the time coordinate and has shown a positive correlation between age and disability risk (Long and Pavalko 2004; Peeters et al. 2013). While life expectancy continues to increase, this positive correlation is worrying. Will the disability risk keep increasing with the extension of life span? Will the survival duration with disability continue to grow? If the answers are yes, long-term care of older people will turn into an "unaffordable burden" for individuals, families, and the society. Alternatively, the process of ADL can vary among elderly with different features, or in different 
periods, while life span increases. Some research has shown that the ageing process will change with the extension of life expectancy and that people can live longer without severe disability (Christensen et al. 2009). Therefore, it is impossible to accurately predict the increased burden of long-term care arising from population ageing, simply due to the change in chronological age, (Schultz and Shoven 2008). In fact, disability is a significant predictor of death (Hirsch et al. 2012), and functional decline usually happens in the last period of life. Hence, the starting point of disability may change with life span, but the end point of disability stays the same. For this reason, it is necessary to trace the disability process back from the time of death in order to analyze the trajectory of ADL decline and find its relationship to remaining survival time. With the known pattern of increase in life expectancy, findings from this study will help to improve accuracy in predicting the disability risk of older people and their need for long-term care in the future.

To study disability risk predicted from chronological age, and to explore contradictions between results from different indictors of functional capacity, this study takes remaining survival time as the time coordinate and ADL scale as the indicator of functional capacity in order to investigate the process of functional decline with ageing. We analyze disability trajectories of functional capacity before death among older people who died in 1998-2014. We also show disparities in disability risk and duration of features of ADL and disability in the elderly according to life span and birth cohort, and explore influences of socioeconomic status, early-life experiences, health behaviors, and living environments on disability trajectories.

\section{Literature review}

\subsection{Change trajectories of ADL}

Functional capacity decreases during the ageing process, and this functional decline can be regarded as an age effect. It has been proved that the association between age and ADL is not linear (Mor et al. 1994; Kaplan et al. 1993), and ADL decline quickly in the last months before death ( $\mathrm{Li} 2005$ ). Scholars have classified the disability trajectories of ADL into different groups according to age, severity, and speed of decline, and have shown c changes in ADL during ageing, while identifying groups of people with high-risk of disability (Stenholm et al. 2015; Zimmer et al. 2012). However, since subjects withdraw from observation before death in these studies, data about change in ADL is censored, and the survival time with disability is unknown. Without taking the final change and time of death into consideration, trajectories based on the living elderly may be unable to correctly reflect the whole process of decline, which may result in errors in the classification and estimated shapes of decline trajectories.

Compared with chronological age, survival duration with disability is of greater significance in assessing the burden of long-term care. Although ADL decrease during ageing, there may be differences in the trajectories of ADL change among older people with different life spans, and individuals of the same chronological age may not show the same ADL trajectory. It has been found that the gains in life expectancy 
in low-mortality countries since the 1970's were mostly in years of life with disability, but not for people with severe disability (Robine et al. 1999). In Europe, the duration of older people's life with severe disability did not increase significantly in 1990-2000 (Cambois and Robine 1996). It can be concluded that, with the extension of survival time, the duration of life with disability may be extended, but that with severe disability may not. The changes mentioned above may originate from the age effect arising from ageing, i.e., the process of ADL change differs among older people who died at different ages. Moreover, we should not ignore the possibility that ADL may benefit from social development, and this is usually expressed in the form of a cohort difference. For example, improvement in medical treatment, health services, and socioeconomic status led to a rapid decrease in the prevalence of disability among the American elderly in 1980-2000 (Schoeni et al. 2008). Disparities in age effects of different cohorts can be defined as a "cohort effect". Since most previous research has focused on cross-sectional analysis at the macro-level, age and cohort effects on ADL decline have not been distinguished clearly.

\subsection{Risk factors of disability in ADL}

Status of ADL and the duration of disability are key to quantifying disability trajectories, and are closely related to the occurrence and development of disability, as well as the recovery and mortality risk of the disabled. Mortality risk of disabled older people is strongly related to the duration of disability and is essential in estimating the length of time that long-term care will be needed. Furthermore, the severity of disability determines the manner and intensity of care delivered to the elderly. Previous research has revealed many risk factors closely related to changes in ADL of the elderly, including socioeconomic status, personal behaviors, nutrition, etc. (Martin and Schoeni 2014). These factors affect the occurrence and development of disability of older people in two ways. The most important contributes through physiological change and acts on functional capacity by changing individuals' intrinsic abilities. A healthy lifestyle, including not smoking and frequent physical exercise, can postpone the decline in ADL, shorten the duration of disability, and reduce the risk of severe disability (Hubert et al. 2002). The second is to change external conditions in which individuals live (Heikkinen 2003), reducing requirements for physical functions and strength in daily activities through improving environments and facilities, thus increasing the chance of carrying out ADL. Some research has shown that improvement in living environments and construction of age-friendly facilities have reduced the disability risk of American older people (Costa 2002). However, the two ways that change the functional status by improving intrinsic abilities and external conditions may differ in their effects on functional capacities. The risk of mild functional disability can be reduced by improving environments and employing assistive facilities, while biomedical intervention can effectively inhibit occurrence of and impairment due to disease, further reducing the risk of severe disability (Manton et al. 2006). A healthy lifestyle has greater effects on deceasing the risk of disability and extending active life expectancy than does reducing the mortality risk (Hubert et al. 2002). Differences between the effects of the two 
ways above mentioned may further increase the heterogeneity of ADL, which may be reflected in differences in the risk and duration of disability.

Improvement in both physical health and external environments are affected by families and the society in which individuals live. Socioeconomic status has significant influence on the functional decline of older people (Yang and Lee 2010). However, this influence may be bidirectional, which leads to uncertainties in trajectories. On the one hand, socioeconomic status acts on the disability trajectory of ADL by influencing material survival conditions, understanding of health information, availability of care service, living environments, etc. (Freedman and Martin 1999; Leigh and Fries 1992). Therefore, older people with higher socioeconomic status usually experience lower risk of disability (Freedman et al. 2008). On the other hand, abundant socioeconomic resources can provide necessary support for disabled older people. Among disabled older-people in the USA, the mortality risk of the highly educated is lower than their less educated counterparts (Manton et al. 1997), so they can live longer with disability. Comparing these two aspects, Melzer et al. (2001) concluded that the effects of socioeconomic status on the risk of disability exceed its influences on the recovery and mortality risk of the disabled elderly, and that a higher incidence rate of disability is the main reason for a higher prevalence of disability among those with lower socioeconomic status. However, Zimmer et al. (2012) have pointed out in their research on Chinese oldest-old that people with different socioeconomic features do not show significant disparity in disability trajectories of ADL. They attribute this phenomenon to the selective effect of death: among older people of lower socioeconomic status; only those who are healthy enough can survive to old age. In their old age, gaps in ADL arising from socioeconomic status will narrow and even reverse. Over all, the influence of socioeconomic status on ADL of older people is realized in two ways: "selection" and "protection". The "selective" effect refers to poor socioeconomic status eliminating the weak through high mortality, which selects for individuals who are strong enough to enter old age and are stable in their ADL; the "protective" effect means that higher socioeconomic status will inhibit the occurrence of and impairment due to disease, and influence ADL through improving lifestyle, living environment, medical treatment and care services, which is reflected in lower risk of disability and longer duration of mild or moderate disability. On the one hand, while the selective effect of death becomes weaker, the survival time with disability of older people will continue to lengthen. On the other hand, the inhibiting effect of a healthy lifestyle and age-friendly environment on the risk of disability goes far beyond influencing mortality risk (Hubert et al. 2002; Fries 2002); it also compresses the survival time with disability. Socioeconomic status can determine which effect wins out.

Life course can further increase heterogeneity in disability trajectories of older people. Some research has found that individuals' childhood experiences have long-term influences on their physical condition in old age and that poverty, disease, and malnutrition are closely related to disability in ADL (Hayward and Gorman 2004; Schoeni et al. 2008). Experiences in early-life affect ADL of old age mainly through the incidence of disability (Freedman et al. 2008). Therefore, cohort differences are greater among those who experienced social instability as juveniles. Also, socioeconomic disadvantages and survival 
advantages that accumulate in the life courses of females will affect their risk of disability and mortality after disability (Denton et al. 2004; Kaneda et al. 2009). Compared with those of males, the incidence rate of disability in females is higher, and their recovery rate and mortality after disability are lower, (Manton et al. 2008; Hardy et al. 2008). It can be inferred that the trajectory of females' functional capacity is different from that of males. However, further research is needed on gender differences in decline trajectories of ADL and their risk factors.

\section{Methodology and data sources}

\subsection{Research design}

This study takes Chinese older people aged 65 and older as the research subjects and employs the group-based trajectory model (GTM) for analysis. ADL is treated as the indicator of functional capacity and the remaining survival time (RST) as the time variable, which reveals disability trajectories of functional capacity before death. We explore age and cohort effects in the process of ADL decline, and analyze differences in the disability trajectories of older people arising from socioeconomic status, early-life experience, health behavior, and living environment.

This study has the following three goals: (1) to quantify disability trajectories of ADL, and show how ADL changes in severity and duration of disability as a function of remaining survival time; (2) to assign older people to groups characterized by their disability trajectories, and compare the probabilities that the elderly who die at different ages and were born in different cohorts belong to these trajectory groups; this will show age and cohort effects; and (3) to analyze how the probability that the elderly with different characteristics belong to different trajectory groups, according to how their socioeconomic status, early-life experience, health behavior, and living environment affects their disability risk and the duration of their disability. The aim is to identify those with higher risk of disability and greater need for long-term care.

Analyses of the data involves three steps. First, we use a group-based trajectory model (GTM) to explore disability trajectories that reflect trends in ADL decline, and to assign older people to different groups according to these trajectories. Second, we introduce age at death and birth cohort into the GTM model as covariates, to analyze their influences on the probability that older people are in different trajectory groups. Third, we add variables representing socioeconomic status, early-life experiences, health behaviors, and living environments to the model, to compare the odds ratio of different disability trajectories among older people that differ in these characteristics. Due to gender differences in severity of ADL disability and survival duration with disability among elderly males and females (Manton et al. 2008; Hardy et al. 2008), two models will be built to conduct the data analyses on females and males separately. 


\subsection{Model for data analysis}

A wide variety of statistical methods are used to study the developmental trajectories that describe the course of an outcome over age or time. Hierarchical and latent curve methodologies are common ways to model population variability in growth with multivariate continuous distribution functions. However, when a population is made up of distinct groups, individual-level development trajectories may not be normally distributed across population members. In this case, the group-based trajectory model (GTM) can be employed. GTM is a special form of finite mixture modeling and is designed to identify groups of individuals that follow similar development trajectories (Nagin 2005). In light of the different disability trajectories of ADL that have been observed in earlier research (Zimmer et al. 2014; Wei et al. 2018), GTM is used in this study.

The number of items in ADL that cannot be carried out is defined as the outcome over time in our disability trajectory model. In order to provide a good fit to the probability distribution of the data, which has more zero values as counts than those under the Poisson distribution, and to avoid underestimating the probability of zero values, the zero-inflated Poisson model (ZIP) is used to accommodate our data.

The GTM with different numbers of trajectory groups and polynomial functions of time was tested in order to identify the one with best performance. In this process, disability trajectory models are estimated for males and females separately. Inferences on the optimal numbers of groups for each gender are made using the Bayesian information criterion (BIC) on the significance of parameter estimates. A model with three type of trajectories was found to be the best fit for our data. Then, polynomial functions of time are included in different combinations to depict trajectory shapes, and the optimum combination, defined by the highest BIC value, is identified as the basic model for males and females separately. Additional predictors measuring individual, socioeconomic, behavioral, or environmental characteristics are introduced into the model to simultaneously estimate trajectory group shapes and the relationship of the predictors to the estimated posterior probabilities of trajectory group membership. The estimated probability of trajectory group membership is assumed to follow a multinomial logit function of the covariates in our most general model.

\subsection{Data sources}

Data is from the Chinese Longitudinal Healthy Longevity survey (CLHLS), for which seven waves have been conducted in 1998, 2000, 2002, 2005, 2008, 2011, and 2014. The interviewees born in 1899-1928 and who died before the last survey in 2014 are selected to be analyzed. CLHLS is a nationwide survey that covered 23 of the 32 provinces of China, representing $85 \%$ of the mainland population. Sampling involved random selection of $50 \%$ of the counties and cities in each of 23 provinces. All the centenarians were interviewed in these locales. For each centenarian, one octogenarian and one nonagenarian living nearby were 
interviewed. Approximately equal numbers of males and females were interviewed in each age decade. For the follow-up surveys in 2000-2011, the sample was supplemented for those who died or were lost to follow-up. Since 2002, older people aged 65-79 were added to the sampling frame with the same sampling method and interview (Zeng 2012). Judging by the reliability and validity of the main health indicators, proxy or non-response rates, rate of internal logical error, and the reliability of the death date, as well as data analysis by many researchers, the quality of data from the CLHLS satisfies the requirements of our research (Gu 2007; Bongaarts 2009). Survey non-response rates in the CLHLS were quite low, about $4 \%$ in each wave. Although the individuals' status of ADL originates from subjective assessments of interviewees, its internal consistency and construct validity with other health indicators are generally good (Gu 2007).

In the seven waves from 1998 to 2014, a total of 42,942 older people aged 65 and above were interviewed, among whom $61.1 \%$ died before the survey in 2014 . Another $14.12 \%$ of the samples were still alive in the survey in 2014 , and $24.78 \%$ were lost to follow-up. Although losing samples may cause inaccuracy in our estimates, the loss rate of CLHLS is relatively low compared with surveys of older people in the USA (Mihelic and Crimmins 1997). Former study reveals that the shapes of ADL trajectories and probabilities of trajectory group membership of dropouts were not substantially different from the results of those being followedup successfully (Zimmer et al. 2012). Of samples who have died, a total of 24,201 persons died at age 105 or below, and 40,984 interview records were collected. Information on these older people is used to analyze the disability trajectories of ADL in the remaining life of older people. Distributions of samples and records in the waves from 1998 to 2011 can be seen in Table 1.

Table 1 Distribution of samples and frequency of interview in 1998-2011 waves

\begin{tabular}{lllllllll}
\hline Baseline interview & \multicolumn{9}{l}{ Number of samples in the waves (\%) } & \multirow{2}{*}{ Total number } \\
\cline { 2 - 7 } & 1998 & 2000 & 2002 & 2005 & 2008 & 2011 & \\
\hline 1998 & 52.22 & 26.11 & 14.00 & 5.26 & 1.84 & 0.57 & 12,296 \\
2000 & - & 51.19 & 30.65 & 12.09 & 4.61 & 1.46 & 8310 \\
2002 & - & - & 55.84 & 25.72 & 12.94 & 5.50 & 8947 \\
2005 & - & - & - & 69.05 & 23.83 & 7.12 & 5644 \\
2008 & - & - & - & - & 78.84 & 21.16 & 5519 \\
2011 & - & - & - & - & - & 100.00 & 268 \\
Total sample & 15.67 & 18.21 & 22.60 & 19.15 & 18.21 & 6.15 & 40,984 \\
\hline
\end{tabular}

Data source: screened and sorted from CLHLS 


\subsection{Measurements}

\subsubsection{Dependent variables}

CLHLS data provide an ADL rating scale that includes six items: feeding, dressing, getting on and off the bed, toileting, bathing, and continence. If an individual needs assistance to carry out a certain activity, he/she will be regarded as being limited or disabled in this activity. In the model we analyze, the number of limitations among the six items serves as an independent variable that ranges from 0 to 6 and reflects severity of disability, from being able to take care of oneself to total disability.

\subsubsection{Time and independent variables}

In addition to ADL, indicators for time and information about the individual are included in the GTM. Individual information covers five domains: demographic characteristics, socioeconomic status, childhood experience, health behavior, and living environment. In the GTM, the time variable does not appear in the regression analysis as an independent variable, but works as a coordinate arranging the dependent and independent variables in time order, and determines their places in the disability trajectories. The remaining survival time (RST) of a person is defined as the gap between the time of interview and death, and on the time axis it reflects the process of ADL decline before death.

We take living area, age at death, and birth cohort as variables that define basic demographic characteristics. Living in a rural or urban area reflects the social environment in which older people reside; age at death and birth cohort are essential to identify the age and cohort effects in the decline of ADL. The main occupations of samples before age 60, and medical services received during old age, denote socioeconomic status; childhood experience tells whether an individual was often hungry and whether he/she was able to obtain timely medical treatment when they were sick; whether the individual exercised frequently or engaged in physical labor are proxies for health behavior; and the availability of a toilet at home before death is used to define the living environment. Apart from the living environment, which reflects living conditions during the last period before death, living area, birth cohorts, socioeconomic status, childhood experience, and health behavior are all long-lasting factors that may affect the disability trajectories of functional capacity. Information about these variables comes from the first interview, so the variables included in GTM are all time-stable covariates.

About 60 percent of the samples live in rural areas. The average age at death of samples exceeds 94 and age at death of females is older than that of males. This is consistent with the expectation that older females' life expectancy is longer than that of males. In terms of the age composition, the oldest-old, over age 80, make up the largest proportions; those born in 1899-1908 account for the highest proportion, $36.39 \%$; those born in 1909-1918 are the second highest, 35.78\%. Most of the samples were nonagricultural workers and soldiers, making up 53\% of the total, while farmers exceed $31 \%$. Occupation distributions of males and females are different. The proportions of male elderly who were farmers or white-collar workers, such as 
technical or management professionals, are much higher than those of females, but the fraction of nonagricultural workers among females is greater that in males.

The samples lived through the period of social instability in China as juveniles, and about $69 \%$ of them were often hungry during childhood; over $70 \%$ could not get timely medical treatment when they were sick. Compared with their situations during childhood, medical resources of interviewees improved greatly during their old age, and over $83 \%$ of them received timely medical treatment in case of illness. 68 percent of the elderly did not exercise frequently, but $81 \%$ had engaged in physical labor. Compared with males, females perform worse in health behaviors, and the proportion of females who ever engaged in regular exercises and physical labor are lower than those of males (Table 2).

\section{Results}

\subsection{Change trajectories of ADL before death}

We use GTM to fit the dynamic of change in number of ADL limitations with RST for males and females separately. We find that there are different disability trajectories of ADL before death for older people. We assume that there might be several

Table 2 Covariate distributions at first interview by gender

\begin{tabular}{|c|c|c|c|}
\hline Characteristics & Male & Female & Total \\
\hline Live in urban $(\%)$ & 38.94 & 37.31 & 37.99 \\
\hline Average age at death & 91.81 & 95.93 & 94.24 \\
\hline \multicolumn{4}{|l|}{ Birth cohort $(\%)$} \\
\hline 1899-1908 & 27.42 & 42.51 & 36.29 \\
\hline 1909-1918 & 41.64 & 31.67 & 35.78 \\
\hline 1919-1928 & 18.57 & 10.55 & 13.86 \\
\hline 1929 year and after & 7.53 & 3.64 & 5.24 \\
\hline \multicolumn{4}{|l|}{ Occupation (\%) } \\
\hline Worker/soldier & 49.80 & 54.72 & 52.69 \\
\hline Farmers, herdsmen and fishermen & 36.81 & 27.75 & 31.49 \\
\hline Technical or management professional & 10.15 & 1.47 & 5.05 \\
\hline Have adequate medical service at present $(\%)$ & 83.81 & 83.35 & 83.54 \\
\hline Not sick at present (\%) & 8.96 & 8.15 & 8.49 \\
\hline Had adequate medical service at childhood $(\%)$ & 30.90 & 27.87 & 29.12 \\
\hline Not sick during childhood (\%) & 20.01 & 19.04 & 19.44 \\
\hline Not hungry during childhood (\%) & 33.16 & 30.07 & 31.35 \\
\hline Exercise regularly $(\%)$ & 41.65 & 25.39 & 32.09 \\
\hline Did physical labor regularly (\%) & 84.52 & 78.73 & 81.12 \\
\hline Toilet at home before death (\%) & 85.99 & 86.89 & 86.52 \\
\hline Sample size & 9976 & 14,225 & 24,201 \\
\hline
\end{tabular}

Data sources: summarized and calculated from CLHLS in 1998-2014 
Table 3 Estimates for disability trajectories of ADL by gender from base models

Description of disability trajectory by survival time (year)

Group 1: remaining Group 2: Disability ris- Group 3: disability rising
stable and in good ing quickly and lasting a slowly and lasting a long condition short time time

\begin{tabular}{llrr}
\hline Males & & $\mathbf{1 9 . 1 2}$ & \\
Percentage in group & $\mathbf{6 6 . 4 9}$ & 1.42 & $\mathbf{1 4 . 3 9}$ \\
Intercept & -2.30 & 0.16 & 1.36 \\
Year & & -0.40 & -0.10 \\
Year squared & & & -0.01 \\
BIC & $-17,487.59$ & & \\
Number of observations 9976 & & $\mathbf{2 1 . 4 2}$ & \\
Females & & 1.45 & $\mathbf{2 3 . 0 1}$ \\
Percentage in group & $\mathbf{5 5 . 5 7}$ & 0.19 & -0.08 \\
Intercept & -1.22 & -0.26 & -0.01 \\
Linear & -0.18 & & \\
Quadratic & & & \\
BIC & $-31,873.73$ & & \\
Number of observa- & 14,225 & & \\
$\quad$ tions & &
\end{tabular}

Data sources: calculated from CLHLS in 1998-2014

The results are significant at $p<0.001$ level (in bold)

linear or nonlinear trajectories reflecting a dynamic relationship between RST and the number of limitations in ADL, and we try to fit these with different combinations of trajectories. After comparing values of the Bayesian information criterion (BIC) from all attempts to fit results, we select an optimal model. Results show that linear and non-linear combinations consisting of three trajectories can accurately describe the process of changing in ADL with RST (see Table 3). Samples are assigned to
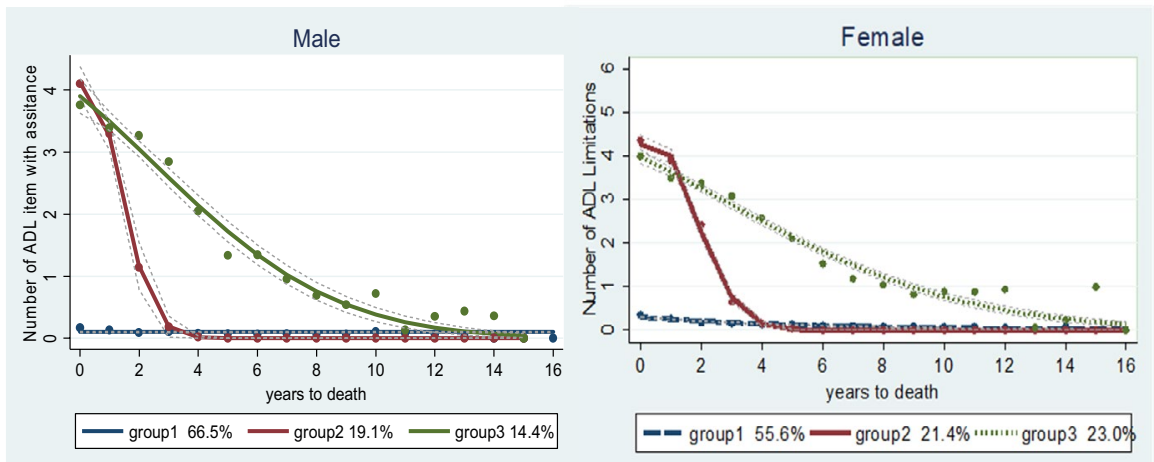

Fig. 1 Predicted number of ADL limitations by years to death and by gender from base models 
three groups characterized by these disability trajectories. One indicator of the model's adequacy is that the average posterior probabilities of membership in each group for all individuals who are mostly likely to belong to that group ranged from 0.70 to 0.88 for females and from 0.76 to 0.90 for males. That this indicator exceeds 0.70 indicates that the performance of our models is acceptable.

As demonstrated in Fig. 1, the ADL of the Chinese elderly declines in different ways that can be characterized by three trajectories. We categorized both male and female experiences in terms of three trajectory groups, all of which are represented by ADL trajectories that show decreasing limitations with RST. Trajectory group 1 is the largest, representing $66.49 \%$ of males and $55.57 \%$ of females; it is characterized by ADL remaining stable and in a fairly good state with few limitations. Trajectory group 2 , which represents $19.12 \%$ of males and $21.42 \%$ of females, starts with a fast decrease in ADL for about three years for males and four years for females before death. Group 3 is the smallest, $14.39 \%$ of males and $23.01 \%$ of females, and shows a slow decline in ADL and a long survival time with disability. Compared to group 3, although the duration of disability in group 2 is shorter, the number of limitations in ADL before death is higher.

\subsection{Gender differences in disability trajectories of ADL}

Disability trajectories of ADL are classified into three kinds and are similar for males and females. However, some gender differences exist in terms of proportional distributions among the elderly which is similar to the former study (Zimmer et al. 2012), as well as the severity and duration of disability. The proportion of males in group 1, whose ADL have good status before death, exceeds that of females in group 1 , while the proportion of females in group 3, whose ADL decline slowly and for a long time, exceeds that of males in corresponding group. Comparing these patterns, the following can be inferred. Although ADL of the elderly in group 1 are relatively good, the linear fitting results for females show that ADL of females in this group decline slightly before death (see Table 3 ). ADL of people in group 2 decline quickly before death, and the duration of the decline among females in this group exceeds that of males, and the number of ADL limitations among females is also higher than among males. ADL for group 3 decline slowly, and the duration of this decline among females also exceeds that among males. We can conclude from these comparisons that both the duration and severity of disability among females exceed those among males and result in longer and higher intensity of long-term care needs for females than for males.

\subsection{Distribution of older people in three groups of trajectory}

We compare age at death, socioeconomic status, childhood experience, health behavior, and living environment of our samples in Tables 4 and 5. We see that disparities exist in the distribution of older people in the three groups. The average age at death for those in group 1 is the lowest and that in group 3 is the 
Table 4 Distributions of elderly females in three trajectory groups

\begin{tabular}{llcc}
\hline Characteristics & Group 1 & Group 2 & Group 3 \\
\hline Live in urban areas (\%) & 35.14 & 38.70 & 42.05 \\
Age at death (mean) & 94.50 & 97.33 & 98.75 \\
Birth cohort (\%) & & & \\
1899-1908 & 38.23 & 46.36 & 51.16 \\
$1909-1918$ & 34.94 & 29.22 & 24.76 \\
$1919-1928$ & 13.50 & 7.47 & 4.86 \\
1929 year and after & 5.19 & 1.83 & 0.77 \\
Occupation (\%) & & & \\
Farmers, herdsmen and fishermen & 26.39 & 31.38 & 29.00 \\
Worker/soldier & 58.36 & 47.77 & 49.70 \\
House worker and others & 13.69 & 19.63 & 19.90 \\
Have adequate medical service at present (\%) & 83.07 & 82.48 & 84.64 \\
Not sick at present (\%) & 8.63 & 8.50 & 6.70 \\
Had adequate medical service in childhood (\%) & 28.27 & 27.76 & 26.87 \\
Not sick in childhood (\%) & 17.73 & 22.40 & 20.28 \\
Was not in hungry in childhood (\%) & 29.76 & 29.92 & 30.99 \\
Exercise regularly (\%) & 27.50 & 22.78 & 21.59 \\
Did physical labor regularly (\%) & 80.44 & 77.92 & 74.82 \\
Toilet at home before death (\%) & 86.40 & 86.57 & 88.35 \\
Size of the sample & 14,225 & & \\
\hline Data sorces: clcule & & & \\
\hline
\end{tabular}

Data sources: calculated from CLHLS in 1998-2014

highest. The proportion of the cohort born after 1909 in group 1 exceeds those in other groups, and the fractions of those born before 1908 are highest in group 3 for females, and group 2 for males.

Occupational distributions of older people in the three groups are also different: proportions of workers and soldiers, farmers, and technical or management professionals are highest in groups 1,2, and 3, respectively. Hence, the three trajectory groups of elderly differ in their socioeconomic resources: the socioeconomic status of those in group 3 is the highest, which is consistent with its higher proportions of urban people and those with adequate medical service resources during old age. For childhood experiences, the proportion of those who were often hungry and could not get timely medical treatment in group 1 is higher than in the other two groups, indicating that people in this group experienced worse socioeconomic status and living conditions as children. The proportion of elderly engaging frequently in physical labor is higher in group 1 than in the other groups. However, the fractions among elderly males and females who exercised regularly are not the same: it is highest in group 1 of females and in group 3 of males.

To further clarify the association between characteristics and disability trajectories of ADL, we introduce these characteristics as covariates in the base model and 
Table 5 Distributions of elderly males in three trajectory groups

\begin{tabular}{llcc}
\hline Characteristics & Group 1 & Group 2 & Group 3 \\
\hline Live in urban areas (\%) & 36.56 & 43.23 & 49.38 \\
Age at death (mean) & 90.84 & 94.17 & 94.71 \\
Birth cohort (\%) & & & 33.86 \\
1899-1908 & 24.20 & 36.83 & 42.97 \\
1909-1918 & 42.63 & 37.15 & 14.62 \\
1919-1928 & 20.76 & 11.99 & 3.37 \\
1929 year and after & 8.99 & 3.82 & 15.52 \\
Occupation (\%) & & & 34.53 \\
Technical or management professional & 9.19 & 11.34 & 45.67 \\
Farmers, herdsmen and fishermen & 35.92 & 41.34 & 85.83 \\
Worker/soldier & 51.99 & 43.28 & 7.31 \\
Have adequate medical service at present (\%) & 83.62 & 83.60 & 31.05 \\
Not sick at present (\%) & 9.20 & 8.82 & 21.93 \\
Had adequate medical service at childhood (\%) & 31.31 & 29.15 & 33.41 \\
Not sick in childhood (\%) & 19.01 & 22.96 & 45.78 \\
Was not in hungry in childhood (\%) & 32.39 & 36.02 & 76.38 \\
Exercise regularly (\%) & 41.55 & 40.05 & 87.63 \\
Did physical labor regularly (\%) & 86.26 & 81.67 & 85.54 \\
Toilet at home before death (\%) & 85.90 & & \\
Size of the sample & 9976 & & \\
\hline
\end{tabular}

Data sources: calculated from CLHLS in 1998-2014

analyze their relationship with posterior probabilities of trajectory group membership provided by GTM.

\subsection{Age and cohort effects on the process of ADL changes}

We introduce age at death and birth cohort into models 1 and 3 as covariates to explore the relationship between these factors and membership in the three disability trajectory groups. Our results show that ages at death of males and females are significantly and positively associated with group 3 versus group 1 membership. Compared with those who died before age 70 , survival past age 90 is positively related to the probability of membership in group 2 or 3 versus group 1, and for centenarians this probability is much higher than for other age groups. Compared with group 2 , group 3 is more likely to conform to the decline in ADL among nonagenarians. Thus, with increasing age at death, the chance of disability increases and the duration of disability is also extended. These are age effects on the changes in ADL during ageing.

Results for models 1 and 3 show that significant disparities exist in the probabilities of four cohorts being assigned to the three trajectory groups. While controlling for age at death, the probability that each cohort is in group 3 versus group 1 is 


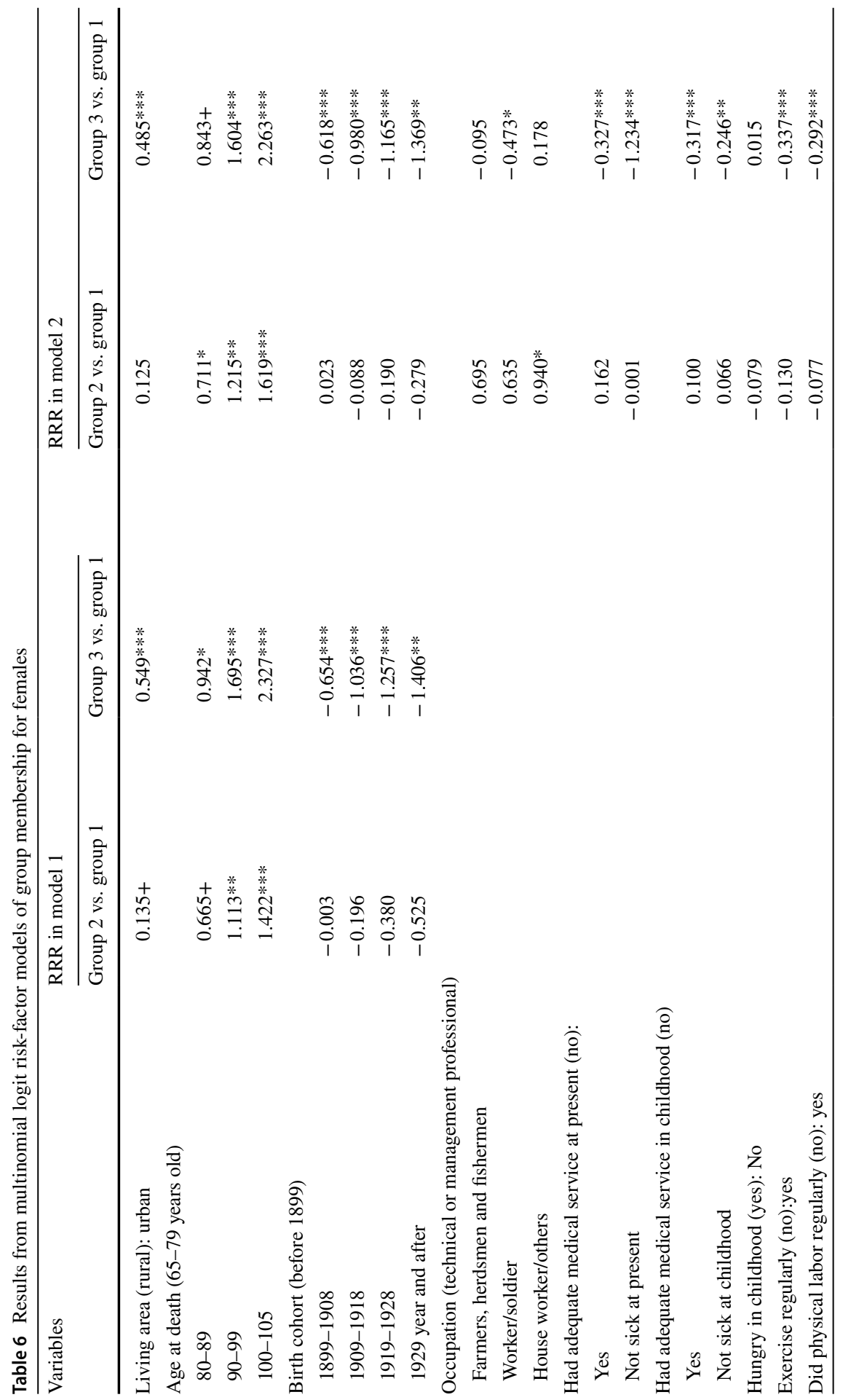




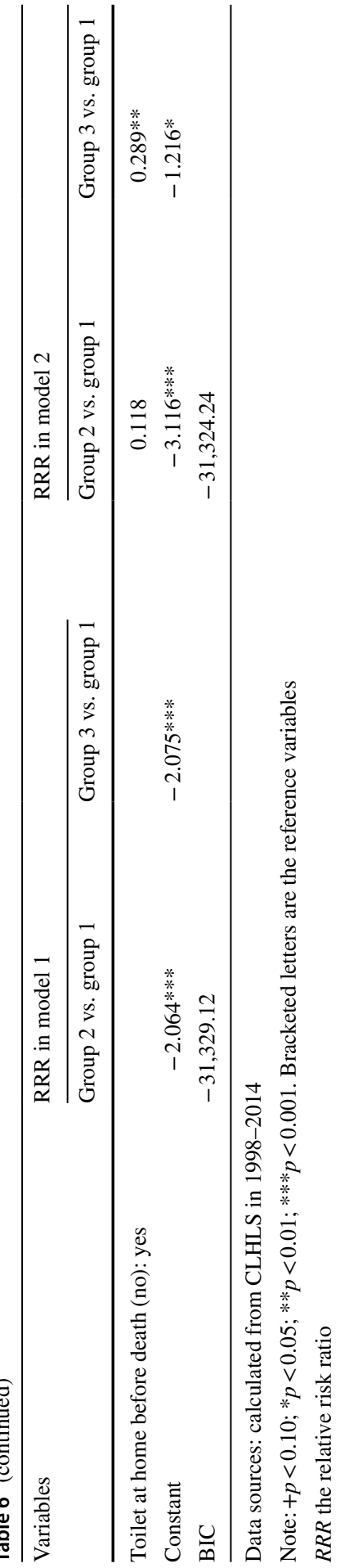


negatively related to the birth year, i.e., the earlier they were born, the more likely they are to be members of group 3 than group 1. Thus, with similar age at death, people born earlier are more likely to experience a major decline in ADL before death and become disabled, while the probability of ADL remaining fairly good is high for those born later. The influences of cohort effects on the process of ADL decline for females are stronger than for males (see Table 6). With later birth years, the likelihood that females experience the disability trajectories of group 3 before death declines faster. By comparing the regression coefficients before and after introducing socioeconomic status, childhood experience, health behavior, and living environment, we find that the introduction of these variables narrows the cohort differences in change trajectories for females, but cohort differences among males become greater. These results suggest that cohort differences in change trajectories of ADL for males and females may be due to different factors.

However, by comparing coefficients of age and cohort in the fitting models, we find that age effects and cohort effects on disability trajectories of ADL are opposite and the former is more powerful. Therefore, with the continuing extension of life expectancy, the decrease in the need for long-term care due to cohort effects will be offset by age effects, which will greatly increase the burden on future families and society for old age support (Table 7).

\subsection{Factors related to the disability trajectories of ADL}

Using baseline models 1 and 3, covariates of characteristics are introduced and produce models 2 and 4, respectively. Regression results show significant heterogeneity in the disability trajectories, and that socioeconomic status, childhood experiences, health behaviors, and living environments are strongly related to the probabilities of the membership in trajectory groups.

Results for model 2 show that, among females, the odds ratio of the elderly being technical or management professionals in group 3 versus group 1 is clearly higher than being workers and soldiers, and the odds ratio of house workers in group 2 versus group 1 is higher than that of other people. Among males, the probability that white-collar workers are in group 3 is higher than for workers, soldiers, and farmers, and the probability that white-collar workers are in group 2 is higher than that of soldiers and workers. In addition, illness is closely related to disability, and it is more likely that older people without serious illness are in group 1, which is characterized by a relatively good ADL status. Results for females in model 3 show that it is less probable for those receiving timely medical treatment to be in group 3, which is characterized by long duration of disability.

Childhood experiences are also associated with disability trajectories of ADL of the elderly. It is more probable that males who were usually hungry were in group 1 and maintained good ADL before death. For both males and females, it is less probable that those who received enough medical care in childhood survive with ADL limitations. Moreover, for females, not getting sick in childhood is negatively 


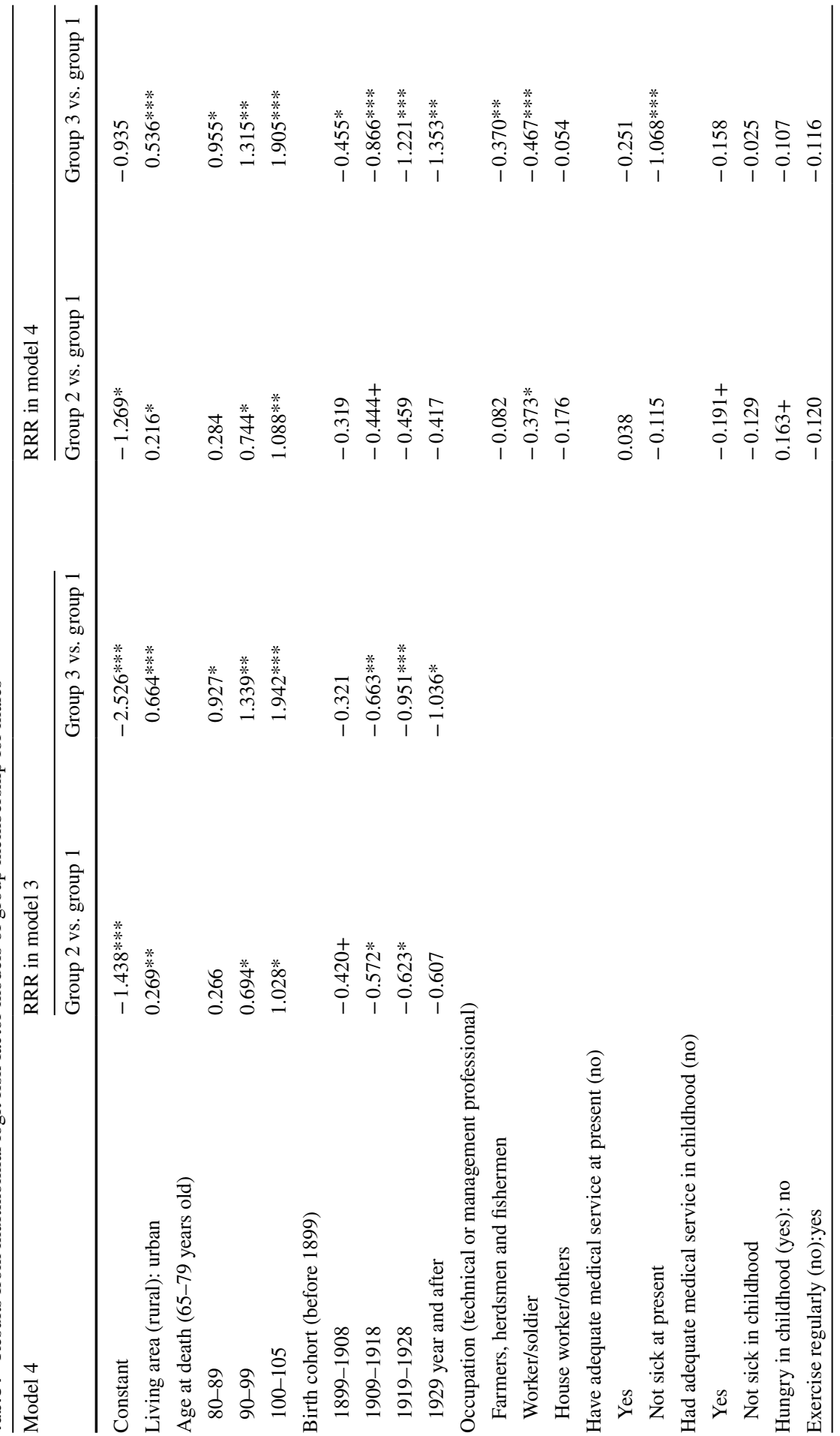




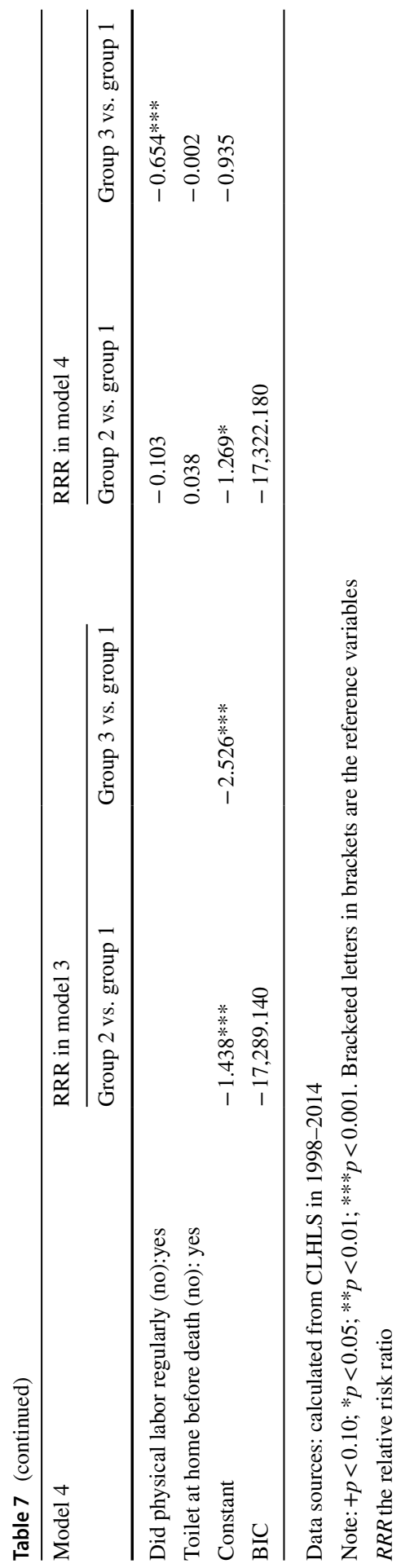


related to the probability of membership in group 3, which has the long duration of disability.

Good health behaviors can reduce the probability that the elderly survive for a long time with disability. For females, model 2 shows that frequent exercise and engaging in physical labor is negatively associated with probability of membership in group 3. Results for model 4 also indicate that males engaging in physical labor are more likely to be assigned to group 1 and maintain good ADL status.

Overall, age at death, birth cohort, socioeconomic status, health behavior, and early-life experience are all significantly associated with the decline in ADL of the elderly and duration of their survival with disability before death. Among the three trajectory groups, the probability of membership in group 3 is influenced by many factors. However, we cannot confirm an effect of living environment. Although it is shown in model 2 that ADL of females with toilets at home are more likely to be in group 3, this may be due to countermeasures for the elderly unable to independently use an outside toilet.

\section{Discussion and conclusion}

We used GTM to fit disability trajectories of ADL before death of Chinese elderly and found three type of trajectories. We also showed the influences of socioeconomic status, childhood experience, health behavior, and living environment on the process of ADL decline. We found that these characteristics affect the probability of membership in the three trajectory groups. The elderly in group 1 maintained ADL in a stable state with few limitations during their remaining survival time, so their need for long-term care due to ADL limitation is the lowest. It is more probable that the elderly who died at younger ages, who were born in later cohorts, and had lower socioeconomic status, such as workers/soldiers and farmers, showed the disability trajectories of ADL of group 1. Good physical health can significantly increase the probability of membership in group 1; for example, individuals not suffering from severe disease and those who have adequate medical resources and healthy behaviors. Group 1 is the most common trajectory of ADL decline among the elderly, especially in males, $66 \%$ of whom fall into this group. The ADL of the elderly in group 2 remain fairly good but decline rapidly in the last period of their lives. At about three years before death, the elderly in this group experience a rapid decrease in ADL. Though their duration of disability is shorter than those in group 3, they have moderate to severe disabilities for most of that time. Group 2 individuals may receive long-term care for about three years because of ADL disabilities, and they need more complex care at greater intensity than others. Most of the elderly in group 2 are females. The oldest-old, agricultural workers, individuals that had good nutrition as children, and those who did not receive timely medical treatment are more likely to fall into the second type of disability trajectory. The ADL of the elderly in group 3 declines slowly over ten years, or even longer, before death and is affected by moderate or severe disability before death. The elderly in group 3 endure the longest survival duration with disability, but experience mild disability for most of the time. Among the three groups characterized by disability trajectories, the elderly 
in group 3 receive care for the longest time and impose the highest burden of care. Females are more likely to belong to Group 3, and it is also more probable that the oldest-old, white-collar workers, and those who did not exercise or experienced severe diseases to belong to this group.

That there are several trajectories in the process of ADL decline of the elderly indicates strong heterogeneity in this process. One main reason is differences in socioeconomic status and health behavior at earlier stages of life. The risk and duration of disability among white-collar workers who have higher socioeconomic status are both greater than those who had other occupations. We deduce that the long duration of disability in white-collar workers originates from the protective effect of higher socioeconomic status. Adequate care, an age-friendly living environment, and high-quality health care can reduce the effect of illness on ADL, decrease mortality risk, and further prolong survival with disability. Our results also show that illness is strongly related to disability, but adequate medical resources can reduce the influence of illness on the decline of ADL. Good nutrition and sufficient medical care in early life reduced the probability that the elderly experience ADL impairment and suffer long period of disability. A possible explanation is that adequate medical resources and good nutrition can reduce the damage that diseases cause to the development of children and improve lifelong physical quality, thus reducing the negative effect of ageing and chronic diseases on ADL. In addition, old females who did not get sick in childhood have lower risk of disability in the long run, which further proves that good physical health in childhood is helpful later in keeping ADL free of disability. For agricultural and home workers of low socioeconomic status, there is a selective effect of death. Only individuals with strong bodies can survive to old age, and this significantly reduces the probability that they experience a long time with disability. Also, they die rapidly after getting sick or disabled because of the shortage of support resources. These people show good and stable ADL before death. However, with decreasing mortality risk for infants and adults, the selective effect through death will gradually be weakened. On the other hand, the protective effect of socioeconomic resources in reducing the risk of disability and prolonging survival with disability will become stronger. Effects of socioeconomic status, early-life experience, and health behavior on the decline of ADL are mainly shown as impacts on the probability of membership in group 3, which is the main determinant of the burden of caring for disabled elderly. We infer that the joint protective effects of socioeconomic status, health behavior, living environment, and other risk factors will play an important role in future needs for long-term care.

Variation in the process of ADL decline is also manifest in the different probabilities of membership in the three groups caused by age and cohort effects. ADL decline due to ageing increases the risk and duration of disability before death among the elderly, and the trajectory of group 3 is more prevalent among nonagenarians. Thus the duration of long-term care needed by the elderly will continue to increase with the extension of life expectancy. However, the extra time that comes from the increase of life expectancy does not entail that it is all spent with disability. For the elderly with the same life span, it is much less likely for those born later to survive for a long time with 
disability, in which case the time for the onset of their disability in ADL is postponed and active life expectancy is extended. As a result, the duration of long-term care is reduced, and the intensity of care needed also decreases. While the influences of age and cohort effects on ADL of older people are opposite, the speed of increase in life expectancy will determine whether the decline in the need for long-term care brought by cohort effects is offset by age effects in the future.

Our study also confirms gender differences in the disability trajectories of ADL and risk factors. From the number of variables significantly related to disability trajectories, the trajectories of females are affected by more factors, and are hence more subject to change than those of males. Although three types of trajectories with similar characteristics have been found, differences exist in patterns and incidence rates of the trajectories between males and females. It is more likely for females to belong to group 3 , characterized by long duration of disability, than for males, while it is more probable that males experience the ADL decline in trajectory 1, which features fairly good and constant ADL status. The above results show that elderly females have a higher risk and a longer duration of disability before death. Probabilities that females belong to the three groups are associated with more factors than those of males, which reflects the instability and high heterogeneity in the process of ADL decline among females. It can be inferred that future growth in the burden of long-term care will be influenced largely by females. Improving socioeconomic status, cultivating healthy behaviors, and providing adequate medical services can effectively improve females' ADL and reduce their need for long-term care.

Although we utilize data from seven waves of the longitudinal survey in our analysis of the disability trajectories of ADL before death among Chinese elderly, our study suffers from a limitation of data and might be improved in future research. First, some samples were still alive in the last wave, so changes of ADL from the last interview to death of these people are not reflected in the data. It can be inferred from the relationship between the factors associated with mortality and those about the risk of disability that the lack of this information may result in lower estimates of the risk and duration of disability among older people. Second, detailed and accurate information of living environments associated with the functional performance is not included in interviews of the survivors, so we could not carry out further analysis on the effect of living environment on ADL.

In conclusion, our research has analyzed the process of ADL decline before death, and quantified three disability trajectories for elderly Chinese males and females, respectively. Trajectories reveal different relationships between ADL and remaining survival time of the elderly. The elderly in the group 3 characterized by long duration of disability in ADL need more long-term care than others, and this is more likely to occur in females and people of high socioeconomic status. The selective effect of death decreases the probability of the elderly dying with disabilities, and the protective effect of socioeconomic resources increases the duration of survival with disability. Age and cohort effects are confirmed in the analysis, and the oldest-old in the early cohort are more likely to survive a long time with disability.

Open Access This article is licensed under a Creative Commons Attribution 4.0 International License, which permits use, sharing, adaptation, distribution and reproduction in any medium or format, as long as 
you give appropriate credit to the original author(s) and the source, provide a link to the Creative Commons licence, and indicate if changes were made. The images or other third party material in this article are included in the article's Creative Commons licence, unless indicated otherwise in a credit line to the material. If material is not included in the article's Creative Commons licence and your intended use is not permitted by statutory regulation or exceeds the permitted use, you will need to obtain permission directly from the copyright holder. To view a copy of this licence, visit http://creativecommons.org/licen ses/by/4.0/.

\section{References}

Bongaarts, J. (2009). Book review on "Zeng Y, Poston DL, Vlosky DA, and Danan G Healthy longevity in china: demographic, socioeconomic, and psychological dimensions". Population and Development Review, 2, 452-453.

Cambois, E., \& Robine, J. (1996). An international comparison of trends in disability-free life expectancy. In R. Eisen \& F. A. Sloan (Eds.), Long-Term Care: Economic Issues and Policy Solutions (1st ed., pp. 11-23). Boston: Springer.

China Research Center on Aging. (2012). Research on situation of urban and rural disabled elderly. Disability Research, 2, 11-16.

Christensen, K., Doblhammer, G., Rau, R., \& Vaupel, J. W. (2009). Ageing populations: the challenges ahead. Lancet, 374, 1196.

Costa, D. L. (2002). Changing chronic disease rates and long-term declines in functional limitation among older men. Demography, 39, 119-137.

Denton, M., Prus, S., \& Walters, V. (2004). Gender differences in health: a Canadian study of the psychosocial, structural and behavioral determinants of health. Social Science and Medicine, 58, 2585-2600.

Evert, J., Lawler, E., Bogan, H., \& Perls, T. (2003). Morbidity profiles of centenarians: survivors, delayers, and escapers. Journal of Gerontology Medical Sciences, 58(A), 232-237.

Fong, J. H., \& Feng, J. (2018). Comparing the loss of functional independence of older adults in the U.S. and China. Archives of Gerontology and Geriatrics, 74, 123-127.

Freedman, V. A., \& Martin, L. G. (1999). The role of education in explaining and forecasting trends in Functional limitations among older Americans. Demography, 36, 461-473.

Freedman, V. A., Martin, L. G., Schoeni, R. F., \& Cornman, J. C. (2008). Declines in late-life disability: the role of early-and mid-life factors. Social Science and Medicine, 66(7), 1588-1602.

Fries, J. F. (2002). Reducing disability in older age. Journal of American Medical Association, 288, 3164-3166.

Gu, D. (2007). General data quality assessment for the 2005 CLHLS wave (CLHLS Technical Report 2007-1). Durham: Center for the Study of Aging and Human Development, Duke University.

Gu, D., \& Zeng, Y. (2004). Socio-demographic effects on ADL dynamics among Chinese oldest-old. Chinese Journal of Population Science Special Issue, 1, 14-22.

Hardy, S. E., Allore, H. G., Guo, Z., \& Gill, T. M. (2008). Explaining the effect of gender on functional transitions in older persons. Gerontology, 54(2), 79-86.

Hayward, M. D., \& Gorman, B. K. (2004). The long arm of childhood: The influence of early-life social conditions on men's mortality. Demography, 41, 87-107.

Heikkinen E (2003) What are the main risk factors for disability in old age and how can disability be prevented? (Health Evidence Network report). Copenhagen: WHO Regional Office for Europe. Retrieved from https://www.euro.who.int/document/E82970.pdf

Hirsch, C. H., Bůžková, P., Robbins, J. A., Patel, K. V., \& Newman, A. B. (2012). Predicting late-life disability and death by the rate of decline in physical performance measures. Age and Ageing, 41, $155-161$.

Hou, C., Ping, Z., Yang, K., Chen, S., Liu, X., Li, H., et al. (2018). Trends of activities of daily living disability situation and association with chronic conditions among elderly aged 80 years and over in China. Journal of Nutrition Health Aging, 22(3), 439-445.

Hubert, H. B., Bloch, D. A., Oehlert, J. W., \& Fries, J. F. (2002). Lifestyle habits and compression of morbidity. The Journals of Gerontology: Biological sciences and Medical Sciences, 57, M347-M351. 
Kaneda, T., Zimmer, Z., Fang, X., \& Tang, Z. (2009). Gender differences in functional health and mortality among the Chinese elderly: testing and exposure versus vulnerability hypothesis. Research on Aging, 31(3), 361-388.

Kaplan, G. A., Strawbridge, W. J., Camacho, T., \& Cohen, R. D. (1993). Factors associated with change in physical functioning in the elderly: a six-year prospective study. Journal of Aging and Health, 5, 140-153.

Katz, S., Ford, A. B., Moskowitz, R. W., Jackson, B. A., \& Jaffe, M. W. (1963). Studies of illness in the aged. The Index of ADL: a standardized measure of biological and psychosocial function. The Journal of the American Medical Association, 185(12), 914-919.

Leigh, J. P., \& Fries, J. F. (1992). Disability in occupations in a national sample. American Journal of Public Health, 82, 1517-1524.

Li, L. W. (2005). Trajectories of ADL disability among community-dwelling frail older persons. Research on Aging, 27(1), 56-79.

Liang, Y., Song, A., Du, S., Guralnik, J. M., \& Qiu, C. (2015). Trends in disability in activities of daily living among Chinese older adults, 1997-2006: The China Health and Nutrition Survey. Journal of Gerontology: Biological Sciences and Medical Sciences, 70, M739-M745.

Liu, J., Chen, G., Song, X., Chi, I., \& Zheng, X. (2009). Trends in disability-free life expectancy among Chinese older adults. Journal of Aging and Health, 21, 266-285.

Long, J. S., \& Pavalko, E. K. (2004). The life course of activity limitations: exploring indicators of functional limitations over time. Journal of Aging and Health, 16, 490-516.

Manton, K. G., Gu, X., \& Lamber, V. L. (2006). Change in chronic disability from 1982 to 2004/2005, as measured by long-term changes in function and health in the U.S. elderly population. Proceedings of the National Academy of Science, 13, 18374-18389.

Manton, K. G., Gu, X., \& Lowrimore, G. R. (2008). Cohort changes in active life expectancy in the U.S. elderly population: Experience from the 1982-2004 National Long-Term Care Survey. Journal of Gerontology: Social Sciences, 63, S269-S281.

Manton, K. G., Stallard, E., \& Corder, L. (1997). Education-specific estimates of life expectancy and age-specific disability in the US elderly population 1982-1991. Journal of Aging and Health, 9(4), 419-450.

Martin, L. G., Feng, Q., Scheoni, R. F., \& Zeng, Y. (2014). Trends in function and activity limitations among Chinese oldest-old, 1998-2008. Population and Development Review, 40, 475-495.

Martin, L. G., \& Schoeni, R. F. (2014). Trends in disability and related chronic conditions among the forty-and-over population: 1997-2010. Disability and Health Journal, 7, S4-S14.

Melzer, D., Izmirlian, G., Leveille, S. G., \& Guralnik, J. M. (2001). Educational differences in the prevalence of mobility disability in old age: The dynamics of incidence, mortality, and recovery. Journal of Gerontology: Psychology Science and Social Science, 56(5), S294-301.

Mihelic, A. H., \& Crimmins, E. M. (1997). Loss to follow-up in a sample of Americans 70 years of age and older: The LSOA 1984-1990. Journal of Gerontology: Psychology Science and Social Sciences, 52, S37-S48.

Mor, V., Wilcox, V., Rakowski, W., \& Hiris, J. (1994). Functional transitions among the elderly: patterns, predictors and related hospital use. American Journal of Public Health, 84, 1274-1280.

Nagin, D. S. (2005). Group-based modeling of development. Cambridge: Harvard University Press.

Ostir, G. V., Carlson, J. E., Black, S. A., Rudkin, L., Goodwin, J. S., \& Markides, K. S. (1999). Disability in older adults 1: Prevalence, causes, and consequences. Behavior Medicine, 24(4), 147-156.

Peeters, G., Dobson, A. J., Deeg, D. J. H., \& Brown, W. J. (2013). A life-course perspective on physical functioning in women. Bulletin of the World Health Organization, 91, 661-670.

Robine, J. M., \& Michel, J. P. (2004). Looking forward to a general theory on population aging. Journals of Gerontology: Biological sciences and Medical Sciences, 59, M590-M597.

Robine, J. M., \& Romieu, I. (1999). Cambois E Health expectancy indicators. Bull WHO., 77, 181-185.

Schoeni, R. F., Freedman, V. A., \& Martin, L. G. (2008). Why is late-life disability declining? The Milbank Quarterly, 86(1), 47-89.

Schultz, G., \& Shoven, J. (2008). Putting our house in order: a guide to social security and health care reform. New York: Norton.

Stenholm, S., Westerlund, H., Head, J., Hyde, M., Kawachi, I., Pentti, J., et al. (2015). Comorbidity and functional trajectories from midlife to old age: the health and retirement study. Journals of Gerontology: Biological Sciences and Medical Sciences, 70(3), M332-M338. 
Vaupel, J. W., \& Yashin, A. I. (1985). Heterogeneity's ruses: some surprising effects of selection on population dynamics. The American Statistician, 39(3), 176-185.

Verbrugge, L. M., \& Jette, A. M. (1994). The disablement process. Social Science and Medicine, 38, $1-14$.

Wei, M., Li, J., \& Wang, H. (2018). Impact of the disability trajectory on the mortality risk of older adults in China. Archives of Gerontology and Geriatrics, 74, 174-183.

Yang, Y., \& Lee, L. C. (2010). Dynamics and heterogeneity in the process of human frailty and aging: Evidence from the US older adult population. Journal of Gerontology: Social Sciences, 65(2), S246-S255.

Zeng, Y. (2012). Towards deeper research and better policy for healthy aging-using the unique data of Chinese Longitudinal Healthy Longevity Survey. China Economic Journal, 5, 131-149.

Zeng, Y., Chen, H., \& Wang, Z. (2012). Analysis on trends of future home-based care needs and costs for elderly in China. Economic Research Journal, 10, 134-149.

Zimmer, Z., Martin, L. G., Jones, B. L., \& Nagin, D. S. (2014). Examining Late-life functional limitation trajectories and their associations with underlying onset, recovery, and mortality. The Journals of Gerontology: Psychological Sciences and Social Sciences, 69(2), S275-S286.

Zimmer, Z., Martin, L. G., Nagin, D. S., \& Jones, B. L. (2012). Modeling disability trajectories and mortality of the oldest-old in China. Demography, 49(1), 291-314.
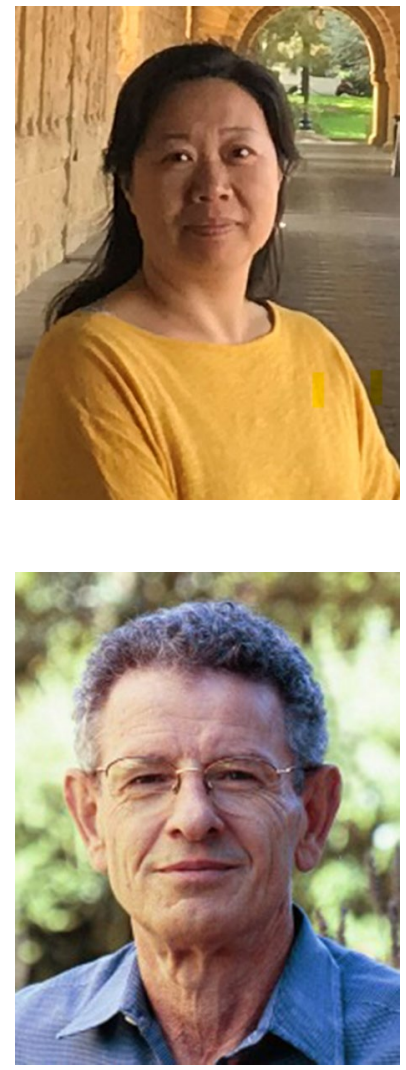

Wenjuan Zhang, PhD is a professor of Institute of Gerontology at Renmin University of China in the School of Sociology and Population studies. Her research focuses on the health of old population and social support for the elderly.

Marcus W. Feldman, PhD is director of Morrison Institute for Population and Resource Studies and Professor of Department of Biological Sciences at Stanford University. In over 620 research publications, he has focused on human genetic and cultural evolution, mathematical biology, and demography of China. 